\title{
Training Novice Users in Bag-Valve-Mask Technique
}

\author{
Adrian A. Matioc, MD
}

William S. Middleton Memorial Veterans Medical Center, Clinical Professor of Anesthesiology, University of Wisconsin School of Medicine and Public Health, Madison, Wisconsin USA

\section{Correspondence: \\ Adrian A. Matioc, MD \\ VA Medical Center \\ Department of Anesthesiology \\ 2500 Overlook Terrace \\ Madison, Wisconsin 53705 USA \\ E-mail: adrian.matioc@va.gov}

Dr. Adrian Matio is the inventor of the ergonomic face mask and receives royalties from King Systems Inc.

Web publication: 26 July 2010
The bag-valve-mask (BVM) ventilation is a core airway management technique for every healthcare provider. Drs. Lin JY, Bhalla N, and King RA are correct in tapping the medical students' pool as a potential resource to carry out lifesaving (one hand) BVM ventilation. ${ }^{1}$ The study demonstrates that the medical students can be rapidly trained for this specific task. This pool of providers may be critical in bioterrorism, pandemic influenza, and/or natural disasters context.

The BVM training success and skill retention also is dependent on the devices used. There is concern regarding the effectiveness and performance of the (conventional-symmetrical) adult face masks. Authors describe "a large variation in both performance and satisfaction between anesthetist with each (face mask) design" ${ }^{2}$ and a need "to improve the design of clear plastic disposable face masks". ${ }^{3}$ The current conventional-symmetrical face mask is not designed specifically for the asymmetrical one hand grip and the airway maneuver built in the "C-E grip" never was validated.

The new asymmetrical ergonomic face mask (ErgoMask ${ }^{\mathrm{TM}}$, King Systems Noblesville, IN) has the left side of the dome larger than the right side and the connector moved to the right and front of the mask (Figure 1). ${ }^{4}$ The specific dome profile allows the left hand to control the whole mask and the fingers 3,4 and 5 to implement a chin lift with head extension: the "chin lift" grip (Figure 2: Picture). The "chin-lift" grip concentrates fingers 3,4 and 5 on the mentum in contrast to the conventional hand position with the $5^{\text {th }}$ finger at the mandibular angle and finger 3 and 4 on the mandibular ramus (Figure 2: Drawing). The measure of an optimal "chin lift" grip is an effective seal (no leak) and airway maneuver (increase of the mentum-sternal distance when cervical spine precautions are not necessary) (Figure 2: Arrow). ${ }^{5}$ The ergonomic face mask can be used also with the two hand technique (jaw thrust) when cervical spine precautions are necessary.

After a brief tutorial, novice airway managers (allied health students) were able to more effectively ventilate a mannequin using the one hand ergonomic face mask compared with the standard face mask technique. Further, performance did not decay over time when subjects were using the ergonomic face mask whereas significant decay did occur with standard mask (Bauman $\mathrm{EB}$, Joffe AM et al). Simulation-based evaluation of an ergonomically designed face mask among novice users. Presented at the International Meeting on Simulation and Healthcare, Phoenix, AZ, January 2010. A face mask designed specifically for one-hand BVM may simplify training, prolong skill retention, and optimize the technique for inexperienced users. Further clinical assessment is needed.

Considering that airway management challenge in the prehospital setting in general and in disaster situations in particular, Drs. Lin $e t$ al should be commended for their focus on this critical topic. 


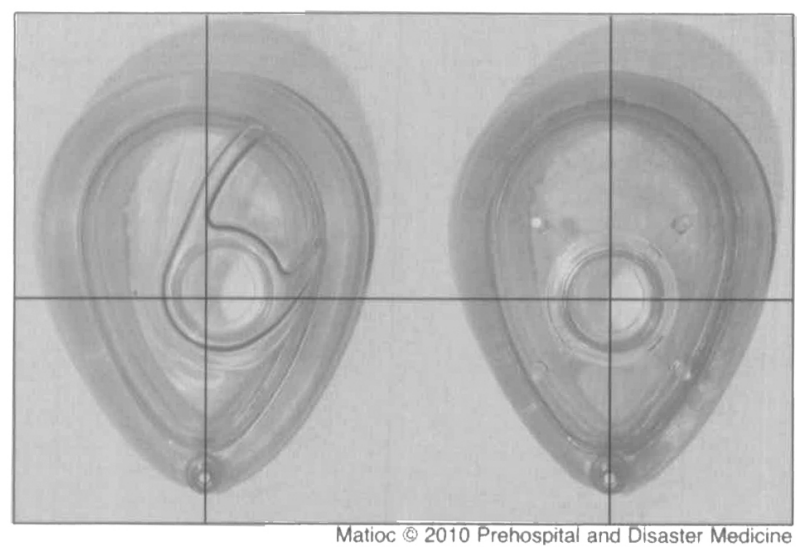

Figure 1-The asymmetrical ergonomic face mask (left) and standard/symmetrical face mask (right)

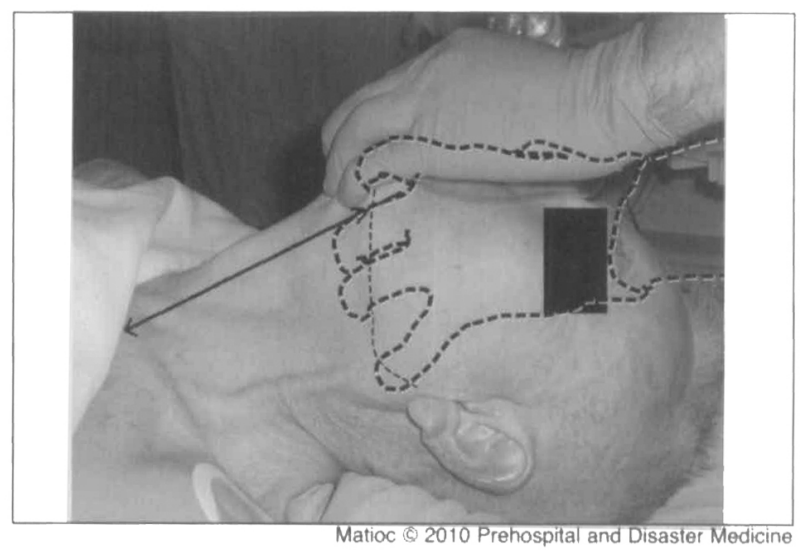

Figure 2-The "chin lift" grip (picture) has full contact with the dome and concentrates fingers 3,4 and 5 on the mentum for a chin lift-head extension. The conventional "C-E grip" (drawing) does not implement a validated airway maneuver. The arrow marks the mentum-sternal distance.

\section{References}

1. Lin JY, Bhalla N, King R: Training medical students in bag-valve-mask technique as an alternative to mechanical ventilation in a disaster surge setting. Prebosp Disaster Med 2009;24(5):402-406.

2. Redfern D, Rassam S, Stacey MR, Mecklenburgh JS: Comparison of face masks in the bag-mask ventilation of a manikin. Eur $J$ Anaestbesiol 2006;23:169-172.
3. Nandalan SP, Waters JH: A comparison of three facemasks used during the induction of general anaesthesia. Eur J Anaesthesiol 2006;23:869-873.

4. The adult ergonomic face mask concept: Historical and theoretical perspectives. J Clin Anesth 2009;21(4):300-304.

5. McGee JP II, Vender JS: Nonintubation management of the airway: mask ventilation. In: Hagberg AC: Benumof's Airway Management. Principles and Practice. Second Edition. Mosby, 2007, pp. 345-370. 